\title{
Study of Tensile Strength Distribution Based on Composite Materials for Aeronautical Engineering
}

\author{
Baojiang $\mathrm{Du}^{1}$, Changqin $\mathrm{Ji}^{1}$, Ping $\mathrm{Chen}^{2}$, Jingmin Guo ${ }^{1}$, Anbo $\mathrm{Sun}^{1} \&$ Xiong $\mathrm{Wei}^{1}$ \\ ${ }^{1}$ College of Mechanical Engineering, University of Shanghai for Science and Technology, China \\ ${ }^{2}$ Shanghai aircraft manufacturing Co., LTD, Shanghai, China \\ Correspondence: Changqin Ji, College of Mechanical Engineering, University of Shanghai for Science and \\ Technology, 516 Jun Gong Road, Shanghai 200093, China. Tel: 86-159-2115-8069. E-mail: \\ jichangqin@126.com
}

Received: February 27, 2012

Accepted: March 15, $2012 \quad$ Online Published: May 1, 2012

doi:10.5539/mas.v6n5p21

URL: http://dx.doi.org/10.5539/mas.v6n5p21

\begin{abstract}
The Tensile strength of an composite materials for aeronautical engineering was tested and its distribution was analyzed based on the two parameter weibull distribution. The maximum normed residual (MNR) test was applied to eliminate abnormal data. A graphical method was used to estimate parameters. Furthermore, the validity of the assumed distribution was examined by the kolmogorov test (KS). The results show that MNR test can be used to exclude abnormal data. It also suggests that the gained two parameter weibull distribution can be used to express the tensile strength and predict its values accurately.
\end{abstract}

Keywords: composite materials for aeronautical engineering, tensile strength, eliminate abnormal data, weibull distribution, kolmogorov test

\section{Introduction}

Composite materials have been developed rapidly. The dosage of composite materials in the airplane structure is growing (Huang C. G. \& Wu G. H., 2002). Its mechanical properties are more and more important in the plane structure design. To arrive at indispensable reference on design and using of the composite materials it is very necessary to predict its mechanical properties distribution.

Generally, the distribution of the composite materials strength obeys weibull distribution, normal distribution, logarithmic normal distribution and so on. This paper uses the tensile strength test data, the research hypothesis assumed that the data obey weibull distribution. The maximum normed residual (MNR) method was applied to eliminate abnormal data, the kolmogorov test (KS) was used to exam the assume distribution.

\section{Test}

\subsection{Test Materials}

The test materials are high modulus fiber reinforced polymer matrix composite laminated boards, produced by an airplane design research institute.

\subsection{Test Sample and Method}

The tensile test references ASTM D3039. The sample is $175 \mathrm{~mm} \times 15 \mathrm{~mm} \times 2 \mathrm{~mm}$ dumbbell specimens. The loading rate is $2 \mathrm{~mm} / \mathrm{min}$.

\section{Test Results and Analysis}

\subsection{Composite Materials Tensile strength Distribution Model}

First, the test data needs a loop check for eliminating abnormal data, then using KS method to inspect hypothesis distribution according to weibull distribution, normal distribution, logarithmic normal distribution of the sequence. The above analysis process is shown in Figure 1. 


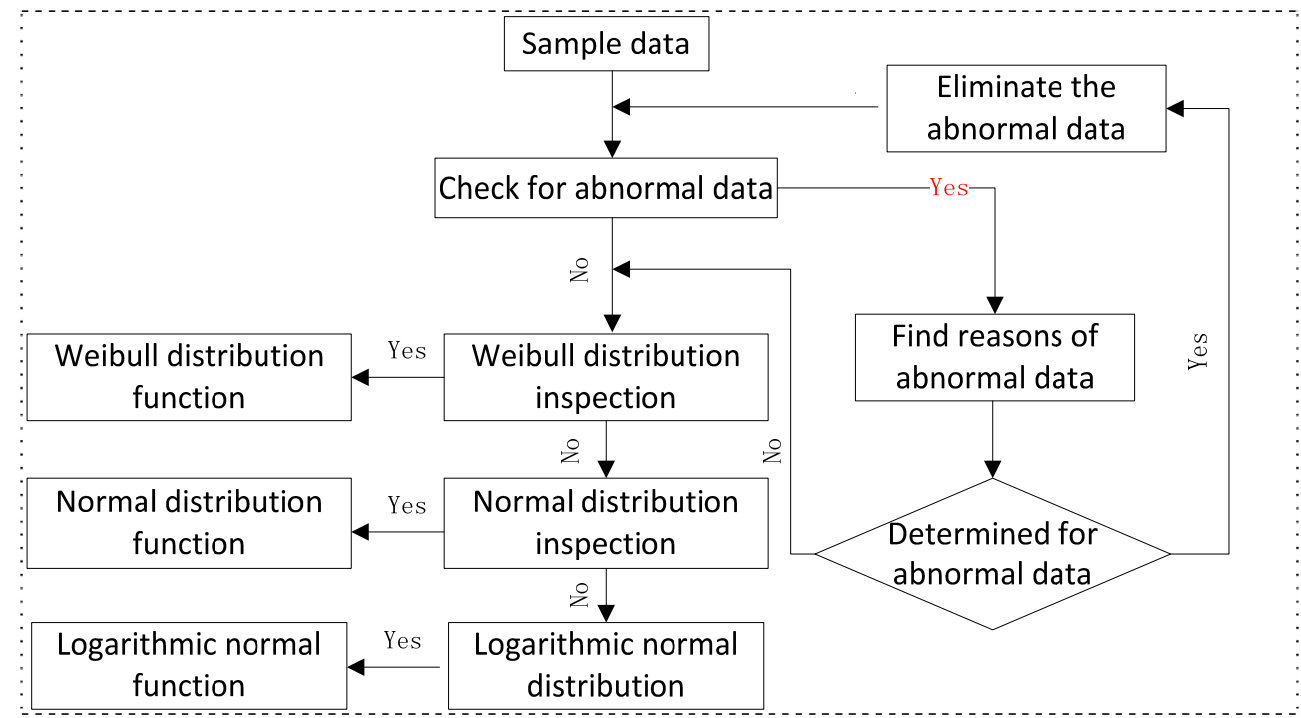

Figure 1. The analysis process for tensile strength distribution of the composite materials for aeronautical engineering

In this article, the composite materials distribution is assumed to be two parameter weibull distribution:

$$
P=F\left(\sigma \mid \sigma_{0}, m\right)=1-\exp ^{-\left(\frac{\sigma}{\sigma_{0}}\right) m}
$$

In the formula (1), parameter $\mathrm{m}$ represents the shape parameter and $\sigma_{0}$ represents the characteristic strength. The larger the parameter $\mathrm{m}$, the smaller the strength dispersion, then the material is more uniform.

\subsection{Eliminating Abnormal Data}

The occurrence of abnormal data is caused by several reasons, such as recording error, using the specimen with defects, wrong environmental conditions. In a group of data, if one data is much lower or higher than the majority of the observed values, it is abnormal. These abnormal data have substantial impact on statistical analysis, so abnormal data must be eliminated before statistical analysis (Shao T. T., Zhang S. L, \& Zhang Y. B., 2008).

In this article, the method MNR is used to eliminate the abnormal data. Relative to sample standard deviation, if the absolute value of the deviation between a data and the sample mean is too large, and the deviation is not caused by chance factors, the data is an abnormal data.

Assume that $x_{1}, x_{2}, \cdots x_{i} \cdots x_{n}$ are the members of the sample, $\bar{x}$ is the sample mean, subscript $i$ denotes the ith $(1 \leq i \leq n)$ member, the letter $n$ denotes the size of the sample. $s$ is the sample standard deviation. When examining abnormal value, make a comparison between MNR statistics and the critical value. MNR statistics can be calculated by the formula (2), the critical value can be calculated by by the formula (3).

$$
\begin{aligned}
M N R & =\frac{\max \left|x_{i}-\bar{x}\right|}{s} \quad i=1,2, \ldots, n \\
C & =\frac{n-1}{\sqrt{n}} \sqrt{\frac{t^{2}}{n-2+t^{2}}}
\end{aligned}
$$

In the formula (3), $t$ is the T-distribution quantile as confidence is $[1-\mathrm{a} / 2 \mathrm{n}]$. $\mathrm{a}$ is 0.05 .

If MNR statistics is less than the critical value $C$, there is no abnormal data in the sample, otherwise, $x_{i}$ which corresponds with the maximum of $\left|x_{i}-x\right|$ is the abnormal data. The examined abnormal data must be eliminated or corrected, then the MNR method is used to inspect the sample until there is no abnormal data. According to the tensile strength test data $(\sigma)$ of the composite, using the above MNR method for screening the test data, the mean of the data $\bar{\sigma}$ is 1239.9 and the standard deviation $\mathrm{s}$ is 1648.7. The corresponding calculation process is as shown in Table 1. 
Table 1 . The process of eliminating abnormal data

\begin{tabular}{ccccc}
\hline Number & $\begin{array}{c}\text { Break strength } \\
\sigma \mathrm{i} / \mathrm{Mpa}\end{array}$ & $\frac{\sigma_{\mathrm{i}}-\bar{\sigma}}{\mathrm{s}}$ & $\begin{array}{c}\text { Corrected Break strength } \\
\text { бi/Mpa }\end{array}$ & Again $\frac{\sigma_{\mathrm{i}}-\bar{\sigma}}{\mathrm{s}}$ \\
\hline 1 & 847.81 & 0.2378 & 847.81 & 0.521 \\
2 & 807.13 & 0.2625 & 807.13 & 1.523 \\
3 & 868.49 & 0.2252 & 868.49 & 0.011 \\
4 & 855.46 & 0.2331 & 855.46 & 0.332 \\
5 & 838.23 & 0.2436 & 838.23 & 0.757 \\
6 & 913.38 & 0.198 & 913.38 & 1.094 \\
7 & 899.87 & 0.2062 & 899.87 & 0.762 \\
8 & 910.76 & 0.1996 & 910.76 & 1.03 \\
9 & 857.81 & 0.2317 & 857.81 & 0.274 \\
10 & 902.69 & 0.2045 & 902.69 & 0.831 \\
11 & 875.53 & 0.221 & 875.53 & 0.162 \\
12 & 821.47 & 0.2538 & 821.47 & 1.17 \\
13 & 795.2 & 0.2697 & 795.2 & 1.817 \\
14 & 820.23 & 0.2545 & 820.23 & 1.2 \\
15 & 8242.6 & 4.2473 & 824.26 & 1.1 \\
16 & 910.69 & 0.1997 & 910.69 & 1.028 \\
17 & 917.86 & 0.1953 & 917.86 & 1.205 \\
18 & 900.42 & 0.2059 & 900.42 & 0.775 \\
19 & 909.86 & 0.2002 & 909.86 & 1.008 \\
20 & 901.8 & 0.205 & 901.8 & 0.809 \\
\hline
\end{tabular}

Description: According to Table 1 calculating results, MNR statistics is 4.2473 on the basis of the formula (3), when the sample degrees of freedom $n=20$, the critical value $C=2.708$, so the fifteenth observational value of the test data is abnormal. The actual data is 824.26 , the MNR method is used again to inspect the test data after the abnormal data is corrected, the mean and the standard deviation of the data were recalculated, the mean is 868.9475 , the standard deviation is 40.6009 , the new calculation result is shown as the last column of Table 1 . The new calculation results indicates there is no abnormal data already.

\subsection{Weibull Distribution Parameter Estimation}

To inspection the assumed two parameter weibull distribution, the estimate values of the shape parameter and the characteristic strength are must be calculated, that is, the parameter estimation is done first (Xiong H. F., 2009). Ranking the tensile strength test data of the composite according to the order from small to large, the test specimen probability fracture is get from the formula (4).

$$
P=F\left(\sigma_{i}\right)=\frac{i-0.5}{n}
$$

$\mathrm{n}$ denotes the times of the total test, $\mathrm{i}$ denotes it is the ith, the time of the test. Taking the logarithm on both sides of the formula (1), The transform result is:

$$
\begin{gathered}
Y_{i}=\ln \{\ln [1 /(1-P)]\} \\
X_{i}=\ln \left(\sigma_{i}\right) \\
B=-m \ln \left(\sigma_{0}\right) \\
Y_{i}=m X_{i}+B
\end{gathered}
$$

As the formula (8) shown, the two parameter weibull distribution function is transformed into a linear relationship. Draw the linear figure between $\mathrm{Xi}$ and $\mathrm{Yi}$, the figure is weibull probability plot (WPP) of the composite. If the regression line is a straight line, the correlation coefficient of $\mathrm{Xi}$ and $\mathrm{Yi}$ is close to 1 , the composite material strength distribution can use the two parameter weibull distribution to describe, otherwise, it 
is not suitable for the two parameters weibull distribution. It is convenient to get the parameters of the two parameter weibull distribution by the parameters $\mathrm{m}$ and $\mathrm{B}$ of the fitting line.

Take tensile strength for example, the two parameters of the weibull distribution were obtained through the fitting line parameter. The Figure 2 and the Table 2 are respectively the WPP of the composite material tensile strength and the main calculation process of weibull parameter estimation.

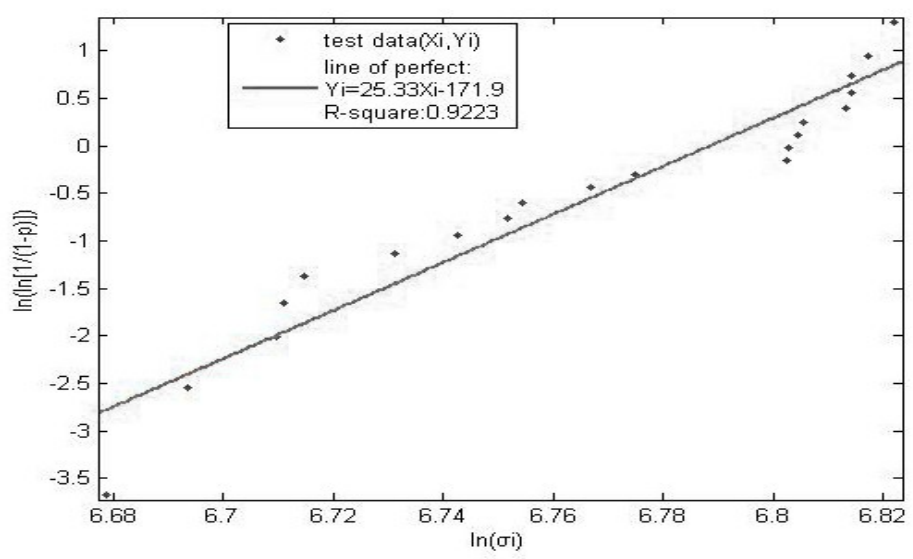

Figure 2. WPP for tensile strength

Table 2. Data to calculate the parameters of weibull distribution

\begin{tabular}{|c|c|c|c|c|}
\hline Number & $\begin{array}{l}\text { Break strength } \\
\text { бi/Mpa }\end{array}$ & $\begin{array}{l}\text { Probably of break strength } \\
P=\frac{i-0.5}{n}\end{array}$ & $Y i=\ln \{\ln [1 /(1-P)]\}$ & $X i=\ln \sigma_{i}$ \\
\hline 1 & 795.20 & 0.025 & -3.6762 & 6.6786 \\
\hline 2 & 807.13 & 0.075 & -2.5515 & 6.6935 \\
\hline 3 & 820.23 & 0.125 & -2.0134 & 6.7096 \\
\hline 4 & 821.47 & 0.175 & -1.6483 & 6.7111 \\
\hline 5 & 824.26 & 0.225 & -1.3669 & 6.7145 \\
\hline 6 & 838.23 & 0.275 & -1.1345 & 6.7313 \\
\hline 7 & 847.81 & 0.325 & -0.9338 & 6.7427 \\
\hline 8 & 855.46 & 0.375 & -0.755 & 6.7516 \\
\hline 9 & 857.81 & 0.425 & -0.5917 & 6.7544 \\
\hline 10 & 868.49 & 0.475 & -0.4395 & 6.7668 \\
\hline 11 & 875.53 & 0.525 & -0.2951 & 6.7748 \\
\hline 12 & 899.87 & 0.575 & -0.1559 & 6.8023 \\
\hline 13 & 900.42 & 0.625 & -0.0194 & 6.8029 \\
\hline 14 & 901.80 & 0.675 & 0.1168 & 6.8044 \\
\hline 15 & 902.69 & 0.725 & 0.2554 & 6.8054 \\
\hline 16 & 909.86 & 0.775 & 0.3999 & 6.8133 \\
\hline 17 & 910.69 & 0.825 & 0.5556 & 6.8142 \\
\hline 18 & 910.76 & 0.875 & 0.7321 & 6.8143 \\
\hline 19 & 913.38 & 0.925 & 0.9518 & 6.8172 \\
\hline 20 & 917.86 & 0.975 & 1.3053 & 6.822 \\
\hline
\end{tabular}

From the above Figure 2 and Table 2, the parameters can be get $\mathrm{m}=25.33, \sigma_{0}=885.7363$. Then the distribution function is: 


$$
P=F\left(\sigma \mid \sigma_{0}, m\right)=\int_{0}^{\sigma} m \sigma_{0}^{-m} t^{m-1} \exp ^{-\left(\frac{t}{\sigma_{0}}\right)^{m}} d t=1-\exp ^{-\left(\frac{\sigma}{885.7363}\right) 25.33}
$$

\subsection{Inspect the Hypothesis Distribution}

The hypothesis distribution inspection is essentially a non parametric test problem (Yu X. H., Zhang L. B., \& Wang C. H., 2007). The performance of the model was evaluated using statistical measures of the maximum distance D. In this paper, KS method is used to inspect the hypothesis distribution of the tensile strength of the composites. KS is based on the cumulative distribution function, compare the cumulative probability gap between the theoretical frequency and actual frequency to find the maximum distance D. Weather the actual frequency distributions is amenable to the theoretical frequency distribution or not is determined by the value of D. The maximum distance D can be calculated by the formula (9).

$$
D=\max [|F(x)-G(x)|]
$$

In the formula (9), $\mathrm{F}(\mathrm{x})$ denotes the empirical distribution function, $\mathrm{G}(\mathrm{x})$ denotes the theoretical distribution function.

Assume that $x_{1}, x_{2}, \ldots x_{n}$ is a set of samples from $F(x)$, and suppose $F(x)$ is amenable to the theoretical distribution, that is to inspection:

$\mathrm{H} 0: \quad \mathrm{F}(\mathrm{x})=\mathrm{G}(\mathrm{x}) \quad \mathrm{H} 1: \quad \mathrm{F}(\mathrm{x}) \neq \mathrm{G}(\mathrm{x})$

Under the given significance level, the critical value $D_{n, \alpha}$ can be looked up when $P\left(D>D_{n, \alpha}\right)=\alpha$. If the maximum distance $D<D_{n, \alpha}$, the null hypothesis is accepted, that is $H 0: F(x)=G(x)$, Otherwise, the null hypothesis is refused.

According to the above mentioned composite materials tensile strength test data, the inspection process is shown in Table 3.

\begin{tabular}{|c|c|c|c|c|}
\hline Number & $\begin{array}{l}\text { Break strength } \\
\text { бi/Mpa }\end{array}$ & $\begin{array}{l}\text { Probably of break } \\
\text { strength } P=\frac{i-0.5}{n}\end{array}$ & $\begin{array}{c}\text { Probably of Weibull } \\
\text { strength } P 0=F\left(\sigma \mid \sigma_{0}, m\right)\end{array}$ & $|P-P 0|$ \\
\hline 1 & 795.2 & 0.025 & 0.0631 & 0.0381 \\
\hline 2 & 807.13 & 0.075 & 0.0906 & 0.0156 \\
\hline 3 & 820.23 & 0.125 & 0.1331 & 0.0081 \\
\hline 4 & 821.47 & 0.175 & 0.1379 & 0.0371 \\
\hline 5 & 824.26 & 0.225 & 0.1493 & 0.0757 \\
\hline 6 & 838.23 & 0.275 & 0.2192 & 0.0558 \\
\hline 7 & 847.81 & 0.325 & 0.2811 & 0.0439 \\
\hline 8 & 855.46 & 0.375 & 0.3392 & 0.0358 \\
\hline 9 & 857.81 & 0.425 & 0.3587 & 0.0663 \\
\hline 10 & 868.49 & 0.475 & 0.4554 & 0.0196 \\
\hline 11 & 875.53 & 0.525 & 0.5255 & 0.0005 \\
\hline 12 & 899.87 & 0.575 & 0.7754 & 0.2004 \\
\hline 13 & 900.42 & 0.625 & 0.7805 & 0.1555 \\
\hline 14 & 901.8 & 0.675 & 0.7933 & 0.1183 \\
\hline 15 & 902.69 & 0.725 & 0.8014 & 0.0764 \\
\hline 16 & 909.86 & 0.775 & 0.8613 & 0.0863 \\
\hline 17 & 910.69 & 0.825 & 0.8675 & 0.0425 \\
\hline 18 & 910.76 & 0.875 & 0.8680 & 0.007 \\
\hline 19 & 913.38 & 0.925 & 0.8867 & 0.0383 \\
\hline 20 & 917.86 & 0.975 & 0.9150 & 0.06 \\
\hline
\end{tabular}

Table 3. Data of kolmogorov test 
The Kolmogorov test statistic $\mathrm{D}=0.2004$, taking $\alpha=0.05$, the critical value $\mathrm{D}_{\mathrm{n}, \alpha}$ can be looked up from the critical value table:

$D_{20,0.05}=0.29403>D=0.2004$

So the null hypothesis is accepted according to the KS method, the composite material tensile strength can use the solved out two parameter weibull distribution to describe.

\subsection{Prediction of the Composite Materials Tensile Strength}

From the above inspection, we can know that the tensile strength distribution of the composite materials can be displayed by the two parameters weibull distribution.

The distribution is shown below on the basis of the formula (1).

$$
f(\sigma)=\frac{d F(\sigma)}{d \sigma}=\frac{m}{\sigma_{0}} \cdot\left(\frac{\sigma}{\sigma_{0}}\right)^{m-1} \cdot \exp ^{-\left(\frac{\sigma}{\sigma_{0}}\right) m}
$$

The mean value of the tensile strength is:

$$
\bar{\sigma}=\int_{0}^{\infty} \sigma f(\sigma) \cdot d \sigma=\int_{0}^{\infty} \sigma \frac{m}{\sigma_{0}} \cdot\left(\frac{\sigma}{\sigma_{0}}\right)^{m-1} \cdot \exp { }^{-\left(\frac{\sigma}{\sigma_{0}}\right) m} \cdot d \sigma
$$

Put the parameters $\mathrm{m}, \sigma_{0}$ into the formula (11), we can get the mean value of the tensile strength is $866.8703 \mathrm{Mpa}$. The measured average value is $868.9475 \mathrm{Mpa}$. The deviation is only $0.24 \%$, the precision is high from the perspective of reliability design (Han G. F., Zhang L. T., \& Cheng L. F., 2007), so using the two parameters weibull distribution to express the tensile strength distribution is reasonable.

\section{Conclusions}

According to the tensile strength test data of the composite materials, first of all, using MNR method to eliminate the abnormal data, and then take the two parameter weibull distribution as model, using graphic method to estimate the parameters, the last, using KS method to inspect the distribution hypothesis and predict the mean value. Make a comparison between the predictive value and the measured average, the deviation is very small, it is $0.24 \%$. So the conclusion is that the tensile strength of composites can be expressed by the two parameter weibull distribution.

\section{Acknowledgment}

The first author gratefully acknowledges the Innovation Fund Project for Graduate Student of Shanghai (No.JWCXSL1102). This work was supported by key grants from Shanghai Science and Technology Foundation (No.10dz1120500).

\section{References}

Han, G. F., Zhang, L. T., \& Cheng, L. F. (2007). Strength Distribution of Silica Fiber and its Minicomposites. Journal of Inorganic Materials, 22(4), 701-705.

Huang, C. G., \& Wu, G. H. (2002). Application of composite materials on helicopter and their development. Composite material life, environment and high technology. Tianjin: Tianjin university press.

Shao, T. T., Zhang, S. L., \& Zhang, Y. B. (2008). Comparison of Two Methods in Eliminating the Excrescent Data. Journal of Modern Electronics Technique, 31(24).

Xiong, H. F. (2009). A Relationship of Dispersity between Fatigue Life and Strength of Composites. Nanjing: Nanjing University of Aeronautics and Astronautics.

Yu, X. H., Zhang, L. B., \& Wang, C. H. (2007). Reliability life analysis of the equipment based on new weibull distribution parameter estimation method. Journal of Mechanical Strength, 29(6), 932-936. 